\title{
Propuesta y diseño de una regla fiscal genérica
}

\author{
A generic fiscal rule: Proposal and design \\ Guido Zack ${ }^{\mathrm{a}}$ y Daniel Sotelsek ${ }^{\mathrm{b}}$ \\ ${ }^{a}$ Universidad de Buenos Aires y Universidad Nacional de San Martín, Argentina \\ ${ }^{b}$ Universidad de Alcalá, España
}

Recibido el 8 de agosto de 2016; aceptado el 5 de diciembre de 2016

Disponible en Internet el 30 de mayo de 2018

\section{Resumen}

Las herramientas esenciales del Estado para cumplir con el objetivo de estabilidad macroeconómica son la política monetaria y la política fiscal. Sin embargo, el avance en términos de reglas de comportamiento ha sido mucho mayor en el caso de la política monetaria. En el presente artículo se pretende hacer un aporte en relación al mecanismo que debería regir una regla fiscal, de forma que fomente un comportamiento contracíclico, sin que ponga en cuestionamiento la solvencia del sector público. Para ello, es fundamental dotar a la regla de flexibilidad, a través de una correcta selección de la base y una cláusula de escape clara y transparente. Una vez descritas sus características deseables, se propone una regla fiscal genérica que debería ser adaptada a cada caso particular, en esta oportunidad el ejercicio se realiza para el caso de España en los años previos a la última crisis. Finalmente, se concluye que una regla fiscal bien diseñada puede ser de mucha utilidad no solo para los objetivos de solvencia y estabilidad, sino también como guía para la discrecionalidad.

Códigos JEL: E32, E62, H60.

Palabras clave: Política fiscal, Reglas, Discrecionalidad, Solvencia, Estabilidad.

\footnotetext{
*Autor para correspondencia.

Correo electrónico: daniel.sotelsek@uah.es (D. Sotelsek)

La revisión por pares es responsabilidad de la Universidad Nacional Autónoma de México. 


\begin{abstract}
The State has two essential tools to achieve the goal of macroeconomics stability, monetary policy and fiscal policy. However, the progress in terms of behavioral rules has been greater in the case of monetary policy. This article aims to make a contribution in relation to the mechanism that should be followed by a fiscal rule to promote a counter-cyclical behavior, without questioning the solvency of the public sector. To do so, the flexibility of the rule is fundamental, through a proper selection of its base and a clear and transparent escape clause. Once described its desirable characteristics, a generic fiscal rule is proposed, which has to be adapted to each particular case. This is done for the case of Spain in the years prior to the last crisis. Finally, it is concluded that a well-designed fiscal rule can be really useful not only for purposes of solvency and stability but also as a guide for discretion.
\end{abstract}

JEL Classification: E32, E62, H60.

Keywords: Fiscal policy, Rules, Discretion, Solvency, Stability.

\title{
Introducción
}

Si queremos revisar la historia de la teoría económica y sus recomendaciones de política, lo mejor es seguir la pista sobre la discusión de cómo debe actuar el Estado frente a las fluctuaciones de la economía. Si bien tanto la política monetaria como la fiscal se consideran esenciales en la posible actuación del Estado, sin duda ha sido la primera el centro de atención durante mucho tiempo. Incluso, hacia finales del siglo XX se impuso la visión que los bancos centrales fueran independientes y que debía existir una regla explícita de política monetaria al estilo de la regla de Taylor. Sin embargo, en el caso de la política fiscal, el tema nunca ha estado muy claro. Más allá de la recomendación o no de tener un presupuesto equilibrado, no hay vocación de coordinar una regla fiscal a seguir por las distintas economías, ni siquiera un consenso sobre si debería existir de la misma forma que la regla monetaria. En ese sentido, este artículo considera que el fenómeno de la regla fiscal, su diseño y su posible implementación, no ha sido del todo analizado, y que los indicadores sobre los cuales se sustenta en muchos casos han tenido fallos que socavaron su credibilidad.

Elaborar una regla de estas características no es una tarea sencilla. Por lo tanto, el presente artículo analiza las características deseables de toda regla fiscal, para luego hacer una propuesta específica y un marco de aplicación práctica. Para ello, en la siguiente sección se incorpora una definición de regla fiscal, indicando los motivos por los cuales su introducción se consideró pertinente, entre los que destacan el incentivo al déficit y a la prociclicidad que poseen los Gobiernos. En la tercera sección, se presenta una serie de trade-offs con los que tienen que lidiar las reglas, tanto en sus dos objetivos más importantes, como en sus características principales. A continuación, se desarrollan las diferentes opciones en relación con el diseño de la regla, en especial en lo que concierne a la base y las cláusulas de escape. En la quinta sección se hace una propuesta de regla, que debería ser adaptada a cada caso particular. Para ello se realiza un ejercicio de aplicación para el caso de España en los años previos a la crisis de 2008, utilizando la estimación del RFE realizada por Zack et al. (2014). Finalmente, se exponen las consideraciones finales. 


\section{Definición, antecedentes y motivos de las reglas fiscales}

Se define como regla fiscal todo elemento legislativo que condicione la conducta de la política fiscal (Debrun et al., 2008: 300) expresada en términos de un indicador de comportamiento a largo plazo, como el déficit, el endeudamiento, o uno de los principales componentes de los mismos (Kopits y Symansky, 1998: 2).

Sus primeros antecedentes datan de mediados del siglo XIX, cuando muchos estados de Estados Unidos asumieron la responsabilidad de adoptar una golden rule ${ }^{2}$ (Buchanan, 1997: 119). Después de la guerra, esta regla de oro se hizo explícita en varios países derrotados (Alemania, Japón e Italia), como parte de sus programas de estabilización. Así, las primeras experiencias tenían como objetivo único y exclusivo la disciplina fiscal (Kopits, 2001: 4-5). Si bien las reglas posteriores mantienen ese objetivo, ya que surgen a partir de las excesivas deudas acumuladas en las décadas de los 70 y 80, también incluyen metas de estabilización y estándares de transparencia para supervisar su efectivo cumplimiento (Debrun et al., 2008: 305).

En esta ocasión, las reglas parecen haber tenido una mayor aceptación, ya que la cantidad de países que hacen uso de ellas para guiar su política ha aumentado de siete en 1990 a ochenta veinte años después (FMI, 2009: 7-9). Claro que esta cifra está influenciada por las reglas supranacionales, presentes no solo en la Unión Europea, a partir de la entrada en vigor del Tratado de Maastrich en 1992 y del Pacto de Estabilidad y Crecimiento en 1997, sino también en las uniones monetarias del Caribe Oriental, del África Occidental y del África Central (Schaetcher et al., 2012: 10-14). De todos modos, en la Unión Europea casi todos los miembros complementan el marco europeo con reglas propias (European Commission, 2009: 87-93).

Ahora bien, ¿por qué son necesarios las reglas fiscales? Hay varios motivos para ello, entre los que se destacan el sesgo al déficit y a la prociclicidad que suele tener el comportamiento discrecional. También juega un papel muy importante el retardo en la implementación de la política fiscal, mientras que la creación de uniones monetarias plantea la necesidad de que venga acompañada, como mínimo, por cierta coordinación fiscal (ver figura 1).

\section{El sesgo al déficit}

Los monetaristas sostienen que tanto la política monetaria (Friedman, 1968) como la política fiscal (Barro, 1974) son incapaces de tener un impacto de largo plazo en la economía real. Es por eso que proponen como política fiscal óptima aquella que mantiene constante la tasa impositiva, es decir, prescinden del uso de medidas discrecionales, dejando la tarea de la estabilización en la política monetaria y en los estabilizadores fiscales automáticos (Friedman, 1948: 247-250; Barro, 1979: 941-950; Lucas y Stockey, 1982).

No obstante, con alguna excepción, algunos autores sí reconocen la capacidad de ambos tipos de políticas para generar efectos en el corto plazo. Por tanto, del mismo modo que la política monetaria posee un incentivo de ser expansiva para fomentar el empleo a costo de una posible mayor inflación (Nordhaus, 1975), la política fiscal tiene un sesgo al déficit (Kydland y Prescott, 1977: 473-474; Buchanan, 1997: 120-123; Halac y Yared, 2014).

\footnotetext{
${ }^{2}$ Phelps (1961) acuñó el término golden rule para referirse a las reglas que obligaban al equilibrio presupuestario en todos y cada uno de los años.
} 


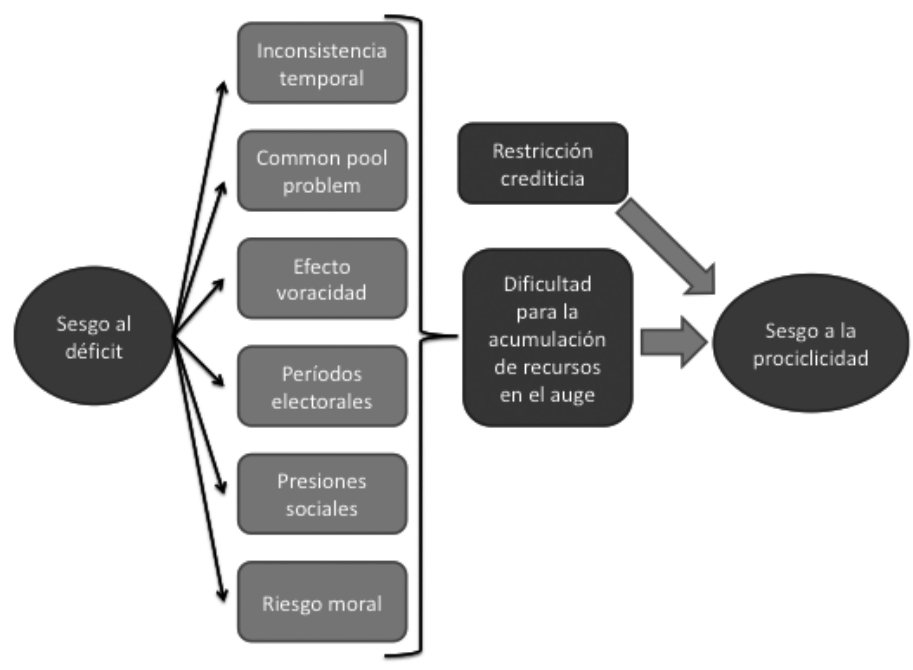

Fuente: Elaboración Propia con base en Debrun et al. (2008).

Este sesgo se ve potenciado en determinadas situaciones, como pueden ser unas elecciones. Dado que los votantes no son conscientes de la restricción presupuestaria intertemporal (Corsetti y Roubini, 1993: 63-70), el Gobierno tiene incentivos a desviarse de la política fiscal óptima, con el objetivo de incrementar el nivel de actividad y empleo, elevando así su imagen positiva (Buchanan y Wagner, 1977: 114-115; Cukierman y Meltzer, 1986; Poterba, 1994: 816$818)^{3}$. El incentivo al desvío no se limita al nivel de gasto público, sino que también incluye su composición aumentando partidas relacionadas con el consumo y reduciendo las vinculadas a la inversión (Rogoff, 1990: 21).

Incluso en momentos no electorales, el denominado common pool problem enseña que determinadas partidas de gasto público pueden ser utilizadas para favorecer a ciertos grupos de interés (Weingast et al., 1981: 650-651; Velasco, 1997: 4).

\section{El sesgo a la prociclicidad}

El common pool problem también genera una tendencia a la prociclicidad. En efecto, en momentos de auge, los ingresos fiscales son abundantes, a la vez que surgen posibilidades de ganancias privadas, lo que incrementa el incentivo al lobby. Así, los recursos fiscales adicionales no se emplean en reducir el déficit o el endeudamiento para estar mejor preparado ante una reversión del ciclo (Lane y Tornell, 1996 y 1998; Tornell y Lane, 1998; Lane, 2002). Este efecto voracidad podría explicar el motivo por el cual tanto en Europa (European Commission, 2001: 63-64) como en otros países desarrollados (Hercowitz y Strawczynski, 2004; Balassone y Francese, 2004) el carácter procíclico se concentra durante los auges, mientras que en las recesiones suele haber un comportamiento más bien acíclico (Manasse, 2006: 15-19).

\footnotetext{
${ }^{3}$ La generación de déficit no solo aumentan sus posibilidades de mantenerse en el poder, sino que - en caso de perder - dejan menos recursos para el siguiente Gobierno (Persson y Svensson, 1989; Alesina y Tabellini, 1990).
} 
Si bien hay evidencia de políticas fiscales procíclicas en diversas regiones del mundo ${ }^{4}$, este sesgo se ve potenciado en los países en desarrollo (Talvi y Vegh, 2005; Alesina y Tabellini, 2005), en parte, porque la restricción crediticia les impide incurrir en déficits fiscales durante la recesión (Kaminski et al., 2004).

Por otra parte, la volatilidad típica de estas economías se refleja en las bases imponibles de los impuestos, impactando finalmente en la recaudación. De esta forma, cualquier intento de suavización inter-temporal del gasto público implicaría incurrir en fuertes superávits durante los auges y en déficits significativos en las recesiones. Sin embargo, al ser economías con amplias necesidades sociales insatisfechas, las presiones para destinar los superávits a tal efecto son muy importantes, por lo que tampoco es tan sencillo generar un ahorro en el auge (Talvi y Vegh, 2005: 177-180).

\section{La pertenencia a una unión monetaria y el retardo en la implementación}

Una unión monetaria implica una única política monetaria y tantas políticas fiscales como miembros tenga la unión. Nada asegura que todas estas políticas sean compatibles con la desarrollada por el Banco Central (Buti et al., 2001). En efecto, hay motivos para sostener que el incentivo al déficit es mayor en estos casos. En primer lugar, la incorporación a un espacio monetario común genera una disminución en los tipos de interés en muchos países, que incentiva el endeudamiento y permite incurrir en elevados déficits fiscales (Buti y Giudice, 2002: 2-4). En segundo lugar, dada la interconexión entre los países y los efectos derrame perjudiciales de una eventual cesación de pagos de un miembro, la posibilidad de ser rescatado genera un riesgo moral que puede animar a las autoridades nacionales a incurrir en déficits mayores (Inman, 1996: 2-3). Tercero, como no tiene a cargo la política monetaria, la miopía política no permite ver los efectos adversos del mayor déficit sobre la inflación (Beetsma y Uhlig, 1999: 553) .

Habiendo cedido la política monetaria (y cambiaria) a un banco central supranacional, surge un desafío muy importante para el diseño de las reglas fiscales: ser lo suficientemente rígidas para evitar déficits excesivos, pero también lo suficientemente flexibles para disminuir la volatilidad.

Incluso los Gobiernos que cuentan con una sólida reputación, por lo que se espera que no presenten los sesgos planteados anteriormente, tienen incentivos para implementar reglas fiscales. El motivo es el tiempo que suele implicar llevar a cabo una medida discrecional en la política fiscal (Kopits, 2001: 8; Wyplosz, 2005: 81-82). En efecto, desde que se identifica la necesidad hasta que la medida tiene efecto pueden transcurrir varios trimestres (Blanchard y Perotti, 1999, 13-16).

\section{Trade-offs de las reglas}

Una vez planteados los posibles costos de la discrecionalidad, surgen una serie de trade-offs en la implementación de las reglas fiscales (ver figura 2).

\footnotetext{
${ }^{4}$ Para una evidencia de una política fiscal procíclica en Europa, ver European Commission (2000: 12-15); para Estados Unidos, ver Taylor (2000: 32-34).

${ }^{5}$ Algo similar ocurre con los gobiernos locales en relación a la administración nacional (Jiménez y Ter-Minassian, 2016; Chamorro Narváez y Urrea Bermúdez, 2016).
} 
Figura 2: Trade-offs de las reglas fiscales

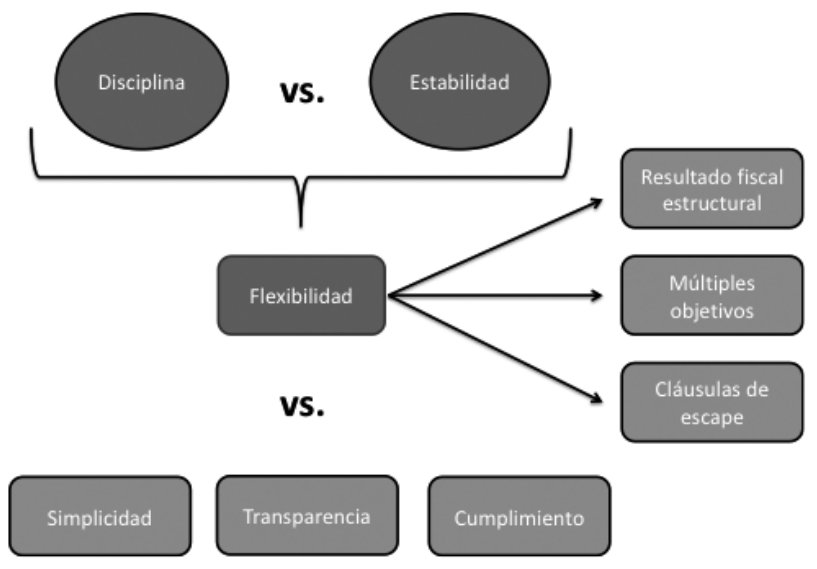

Fuente: Elaboración propia con base en Kopits y Symansky (1998).

El primero es el que se produce entre los objetivos de disciplina y estabilidad. Esto se debe a que, si bien una política fiscal restringida sería efectiva en términos de una mayor solvencia, también tendría una menor reacción ante las fluctuaciones del ciclo económico (Eichengreen, 1992: 30-32; Poterba, 1994: 812-816; Alt y Lowry, 1994: 823-824; Bohn e Inman, 1996: 40-46; Lane, 2003: 5-6).

No obstante, todos estos estudios analizan una sola cara de la moneda, pues solo consideran la posibilidad de que las reglas fiscales restrinjan el carácter contracíclico de las políticas. Por el contrario, también existe la posibilidad de que el comportamiento del sector público tienda a ser procíclico, por lo que imponerle límites puede mitigar un factor adicional de volatilidad (Buchanan, 1997, 127-129; Debrun et al., 2008: 302-303; Alesina y Bayoumi, 1996). Incluso otros autores llegan a la conclusión de que las reglas no solo mejoran el resultado fiscal, sino que también reducen la volatilidad macroeconómica, negando la existencia del trade-off (Ayuso-i-Casals et al., 2006: 687-691; Manasse, 2005 y 2006). Por su parte, Fatás y Mihov (2005) reconocen que la introducción de restricciones, por un lado, limita la respuesta de la política fiscal ante shocks exógenos, aumentando la volatilidad. Pero, por otro, se genera una política fiscal más estable, lo que compensa con creces el efecto anterior.

A partir de lo anterior, se puede decir que el efecto de las reglas sobre la volatilidad no está predefinido y dependerá del objetivo que se persiga con la política fiscal: en caso de realizarse sobre un carácter predominantemente contracíclico, entonces las limitaciones podrían acentuar la inestabilidad, en cambio, si la evolución de la política fiscal es procíclica, las restricciones ayudan a una mayor estabilidad.

No obstante, hay un factor más a considerar. El sesgo al déficit puede llegar a poner en duda la solvencia del Estado (Buchanan, 1997: 120-123). En ese caso, se dificulta seriamente el acceso al mercado de capitales, impidiendo muchas veces la realización de una política fiscal contracíclica, especialmente durante las fases descendentes (Aizenman et al., 1996; Gavin y Perotti, 1997; Catao y Sutton, 2002; Kaminski et al., 2004). 
De todos modos, una forma de combinar el efecto positivo de la regla sobre la disciplina, sin limitar la capacidad contracíclica, es dotar a las reglas de flexibilidad. Hay varias formas de lograr que una regla sea capaz de reaccionar frente a los movimientos de ciclo económico. La primera es establecer la restricción sobre un indicador de largo plazo, como el resultado fiscal estructural (RFE), en lugar del resultado fiscal observado (RFO), dejando así operar los estabilizadores automáticos (Kopits y Symansky, 1998: 24-25). Otra forma, como se verá más adelante, es aplicar la regla a más de un indicador fiscal (Ter-Minassian, 2010: 11-12; Schaechter, et al.2012: 15-16) o bien hacer variar el resultado fiscal objetivo junto con el ciclo económico.

Pero aun las reglas flexibles podrían generar restricciones contraproducentes en términos cíclicos en situaciones excepcionales de fuertes shocks externos. Para estos casos, existen las llamadas cláusulas de escape, que definen las condiciones que deben darse para que el funcionamiento de la regla se suspenda temporalmente y puedan sobrepasarse los límites por ella impuestos. Estas condiciones, así como aquellas que restablezcan el funcionamiento de regla en caso de suspensión temporal, deben ser definidas de antemano en forma explícita, así como de sencilla supervisión.

En definitiva, una regla lo suficientemente flexible podría sortear el eventual trade-off entre solvencia y estabilidad. No obstante, la menor rigidez de las restricciones plantea un gran desafío al diseño de la regla y un nuevo trade-off, pues introducir flexibilidad en la regla necesariamente implica hacerla menos simple y transparente, lo que a su vez dificulta su efectivo cumplimiento (enforcement $)^{6}$.

Una regla flexible pierde simplicidad básicamente por el hecho de tener que ser aplicada a más de un objetivo, porque los objetivos sean más complejos y por la inclusión de las cláusulas de escape. Más aún si estos objetivos pasan de ser indicadores observados, como el gasto, ingreso, resultado fiscal o deuda, a indicadores estimados, como el RFE.

Por su parte, la flexibilidad provoca una demanda mucho mayor en términos de transparencia (Cangiano, 1996). Si bien la publicación de la información relevante de las cuentas fiscales en tiempo y forma es siempre fundamental (Kopits y Craig, 1998: 7-9), esta tarea se vuelve más exigente al tener que hacerlo con indicadores no observados, sino estimados, e incluso proyectados (Buti et al., 2003: 20-21).

Finalmente, dado que la simplicidad y la transparencia son esenciales para la supervisión de la regla y su efectivo cumplimiento (Kopits y Craig, 1998: 19), la mayor exigencia y el eventual deterioro de estas características podría afectar al enforcement y así a la efectividad de las restricciones en la obtención de sus objetivos de solvencia y estabilidad (Tanzi, 1993).

\section{Diseño de las reglas fiscales}

Teniendo en cuenta los sesgos al déficit y a la prociclicidad de la política fiscal discrecional, así como las características deseables de las reglas para corregirlos (flexibilidad, simplicidad, transparencia), en lo que sigue se desarrollan diferentes opciones acerca del diseño de las reglas fiscales en relación con su indicador base y las cláusulas de escape.

\footnotetext{
$\overline{{ }^{6}}$ Trabajos recientes han eliminado la simplicidad como rasgo característico y necesario de las reglas, dado que, si bien es algo deseable, parece no ser posible bajo las particularidades del mundo actual (Schaechter et al., 2009: 5).
} 


\section{La base de la regla}

La base de la regla se refiere al indicador sobre el cual se efectúa la restricción numérica (Ter-Minassian, 2010: 10). Este aspecto reviste suma relevancia, ya que su elección condiciona la respuesta de la regla en relación con la preservación de la solvencia y el mantenimiento de la estabilidad (Bohn e Inman, 1996: 30-34). Asimismo, se debería tener en consideración cuán controlable es por el Gobierno, si es capaz de otorgar una guía operacional en todo momento del ciclo y la facilidad en su supervisión (FMI, 2009: 4 y 20). Los candidatos más frecuentes son el nivel de endeudamiento, el gasto, los ingresos y el resultado fiscal.

\section{El nivel de endeudamiento}

Si el objetivo es asegurar la solvencia del Estado, el primer indicador al que se hace alusión es la deuda pública. Al prohibir que el nivel de endeudamiento supere un porcentaje determinado del PIB, se condiciona la evolución del resultado fiscal y, por lo tanto, también de los ingresos y gastos públicos. Además, una regla de estas características es simple, transparente y fácil de comunicar.

Sin embargo, como único instrumento, tiene el inconveniente que le lleva un tiempo ser influenciada por las modificaciones en las otras variables fiscales, por lo que no restringe a la política en el corto plazo, a la vez que es afectada por factores que no están al alcance del Gobierno, como el tipo de interés (FMI, 2009: 21). Del mismo modo, no genera ningún tipo de guía cuando la deuda se sitúa por debajo del límite. Por último, ante shocks externos capaces de deteriorar de forma significativa el nivel de actividad y los ingresos públicos, el déficit puede generar un incremento del cociente de deuda, obligando a la realización de ajustes, en un claro comportamiento procíclico (Schaechter et al., 2012: 7).

\section{El gasto}

El gasto público total, primario o corriente es una variable definida de forma casi perfecta por el Gobierno (en particular el primario), a la vez que provee de una guía operacional en todo momento y es fácil de supervisar (FMI, 2009: 21). La restricción se basa en limitar la tasa de crecimiento nominal o real, o bien su participación en el PIB.

El condicionamiento al comportamiento cíclico se ajusta mejor cuando se utiliza como referencia la tasa de crecimiento. En efecto, un límite a la variación anual del gasto impone una restricción a un eventual comportamiento procíclico durante el auge o cuando los ingresos se ven incrementados por la evolución de alguna variable en particular (precios de las materias primas, inversión en vivienda, etc.). Al mismo tiempo, permite una acción expansiva en la recesión, aunque limitada.

La carencia principal de este tipo de regla es que no está directamente relacionada con la solvencia, dado que no incluye objetivos de ingresos (Schaechter et al., 2012: 8-9). Así, por un lado, no es capaz de influir sobre el nivel de endeudamiento. Por otro, no parece ser una buena opción en el caso de sistemas fiscales no maduros, ya que se espera que los ingresos públicos aumenten junto con la modernización del Estado y los gastos, en este contexto, bien pueden aumentar a un ritmo similar al de los ingresos sin poner en cuestionamiento la solvencia del sector público (Machinea, 2012: 76). 


\section{Los ingresos}

Una regla con base en los ingresos públicos suele imponer límites máximos y/o mínimos en la presión fiscal. En muchas ocasiones son muy difíciles de cumplir, ya que los ingresos fiscales - a diferencia de los gastos - no están bajo la órbita directa del Gobierno por ser muy dependientes de las variaciones del nivel de actividad (Buti et al., 2003: 16). Incluso, como la elasticidad puede ser mayor que uno, imponer un piso o un techo a la presión fiscal puede limitar el carácter contracíclico del sistema tributario, siendo contraproducente en términos de estabilidad (Schaechter et al., 2012: 9). Asimismo, al dejar de lado los gastos, son también reglas parciales, por lo que son incapaces de asegurar comportamientos responsables en términos de solvencia.

Otros tipos de reglas sobre los ingresos son aquellas que obligan a destinar una parte de la recaudación a un fin específico, muchas veces a generar un ahorro durante los auges bajo la forma de fondos soberanos. Suelen estar relacionados con la presencia de recursos no renovables, por lo que - además de la estabilidad - el objetivo es mejorar la equidad intergeneracional (Jiménez y Tromben, 2006).

\section{El resultado fiscal}

Después de analizar las limitaciones de las reglas sobre las variables anteriores, de cara a los objetivos de solvencia y estabilidad, surge la necesidad de actuar sobre un indicador más completo como es el resultado fiscal (Sacchi y Salotti, 2015). En efecto, su cálculo incorpora tanto los gastos como los ingresos, mientras que su comportamiento condiciona la evolución de la deuda. Así, un resultado fiscal equilibrado a lo largo de todo el ciclo, hace que los gastos se ubiquen en torno a los ingresos, sin generar variaciones significativas en el nivel de deuda, lo que en principio aseguraría la solvencia (Tapia, 2003: 39-41).

Asimismo, un límite sobre el resultado fiscal en términos del PIB es relativamente sencillo de supervisar, a la vez que provee de una guía constante en relación con la dirección que debería seguir la política. Por su parte, el indicador es suficientemente controlable por parte del Gobierno, aunque hay cierta dificultad relacionada con los componentes cíclicos y con los pagos en concepto de intereses (FMI, 2009: 20-21).

No obstante, una regla con esta base no contempla ciertos elementos contracíclicos, que a su vez pueden provocar inconvenientes relacionados con la solvencia. En caso de que el objetivo sea fijo, es decir, no se modifique en función de la fase del ciclo, de cumplirse la restricción cerca del límite durante el auge, es altamente probable que se incumpla una vez que se desacelere la economía, como consecuencia de la caída en los ingresos. De esta forma, para volver a cumplir con la regla, en la recesión el Gobierno se vería obligado a consolidar el resultado fiscal, en una clara acción procíclica (Manasse, 2005: 8-11).

Una opción para mitigar la ausencia de elementos contracíclicos, es aplicar el objetivo sobre el indicador estructural (Ter-Minassian, 2014; Bova et al, 2014). Al hacerlo, se excluyen del campo de acción de la regla los estabilizadores automáticos (Ter-Minassian, 2010: 8-11). Sin embargo, como se ha visto a partir de la crisis de 2008, este indicador no es satisfactorio a la hora de identificar los ingresos producto de la revalorización de activos. Para ello es de suma utilidad desarrollar un indicador estructural que sí considere estos efectos, como el desarrollado por Zack et al., (2014) ${ }^{7}$.

\footnotetext{
${ }^{7}$ Para mayores detalles en relación a la metodología de cálculo del resultado fiscal estructural ver el Anexo I.
} 
Pero incluso si se desea potenciar el efecto estabilizador de la política fiscal, sin necesidad de acudir a cláusulas de escape ni salirse de la regla, se puede plantear algún tipo de mecanismo en el que el nivel objetivo vaya variando a lo largo del ciclo. Una posibilidad es que el objetivo sea un porcentaje fijo del Output Gap (OG). Así, durante los auges, momento en el que el PIB se sitúa por encima del potencial, el RFE objetivo sería positivo. Por el contrario, en las recesiones, cuando el OG es negativo, la misma regla permitiría incurrir en un déficit estructural.

\section{Combinación de bases}

Otra alternativa para abarcar tanto el objetivo de solvencia como de estabilidad es elaborar una regla que combine diferentes bases. Las combinaciones más frecuentes son restricciones sobre el gasto o el resultado fiscal, con límites sobre el nivel de endeudamiento.

Como se ha visto anteriormente, el nivel de endeudamiento es un buen indicador para incentivar actitudes más responsables en términos de solvencia, pero en muchas ocasiones no genera ningún tipo de guía para la política fiscal, a la vez que no implica restricciones importantes en términos cíclicos. Por su parte, el gasto es útil para fines de estabilidad, especialmente cuando se limita su tasa de variación, pero al no contemplar los ingresos es incapaz de actuar de forma global sobre la solvencia. Así, una combinación de ambos indicadores podría incluir los dos objetivos finales, sin generar una regla de gran complejidad y manteniendo su transparencia (Schaechter, 2012: 9).

La limitación principal de esta combinación es que sigue sin considerar de forma directa los ingresos. Una forma de incluir los ingresos de modo más directo es combinando el objetivo deuda con otro de RFE (Ter-Minassian, 2010: 11-12). Nótese que esta combinación es la elegida originalmente por la Unión Europea para sus miembros, aunque por la experiencia reciente, en muchos casos, sin los resultados esperados.

Incluso, podría plantearse una regla en la que el objetivo de la base fluctúe junto con el ciclo, de forma que se fomente el efecto estabilizador. Por su parte, el nivel de endeudamiento, junto a la posible existencia de pasivos contingentes, podría definir el valor sobre el cual variaría el nivel objetivo. En el caso de un país poco endeudado y sin pasivos contingentes, que quiera mantener el nivel de deuda o incluso incrementarlo, este valor podría ser cero o negativo, respectivamente (Blanchard et al., 1990: 10-12; Cuddington, 1997: 6-8). Pero en un país cuya deuda se ubique por encima del máximo permitido, o bien en el caso de que exista una probabilidad real de tener pasivos contingentes (Tapia, 2003: 39-41), este valor podría ser positivo y mayor cuanto más sobrepasado se encuentre sobre el límite.

La idea básica de diseñar una regla sobre un objetivo de RFE fluctuante con el ciclo, dotada de flexibilidad y tener en cuenta el nivel de endeudamiento y los pasivos contingentes, es reducir al máximo posible la necesidad de suspender temporalmente la regla. Sin embargo, ningún diseño puede ser tan completo como para considerar ex-ante todos los escenarios posibles que puedan surgir. Así, siempre deben incluirse cláusulas de escape en la regla.

Esto plantea un importante desafío en el diseño, relacionado con la identificación de esos momentos excepcionales. Un método de identificación efectivo es esencial para permitir el uso de la flexibilidad e impedir los intentos de eludirla simplemente para obtener beneficios de corto plazo, sin consideraciones sobre la solvencia de largo. Asimismo, una vez tomada la decisión de suspenderla temporalmente, se debe especificar el retorno así como el tratamiento de las desviaciones acumuladas (FMI, 2009: 26). Como se verá en el siguiente epígrafe, la regla 
genérica propuesta y su aplicación para el caso Español tienen diseñada una cláusula de escape (ecuaciones 1 y 2). La idea básica es condicionar la desactivación y posterior reactivación a un valor determinado (en este caso del OG) a definir por los gobiernos según cada caso particular.

\section{Propuesta genérica de regla fiscal y aplicación al caso de España}

Habiendo desarrollado las motivaciones que fomentaron la introducción de reglas fiscales, sus características deseables, sus posibles trade-offs y las alternativas en relación con su diseño, a continuación, se propone un modelo genérico de regla.

La regla fiscal propuesta plantea su indicador objetivo en una combinación de bases ya mencionada previamente: el RFE y el nivel de endeudamiento. El primero se utiliza por ser el indicador más completo, en el que se incluyen tanto los ingresos fiscales como los gastos. En particular, se hace uso de su versión estructural, de manera que se da un primer nivel de flexibilidad, al dejar actuar libremente a los estabilizadores automáticos.

Adicionalmente, para acentuar su flexibilidad y su capacidad estabilizadora, se propone que la base objetivo fluctúe junto con el ciclo. La opción más simple e inmediata para ello es que las limitaciones al valor del RFE sean un porcentaje fijo positivo del OG. Así, cuando la economía se encuentra en una fase de auge, el objetivo de RFE será mayor de cero, generando un ahorro. En cambio, en una parte contractiva del ciclo, el OG es negativo y, por tanto, también el RFE objetivo, permitiendo un fomento a la demanda por parte del sector público (figura 3).

El objetivo de solvencia implica que las oscilaciones cíclicas del RFE se centren en cero, es decir, se plantea un equilibrio presupuestario a largo plazo. Sin embargo, dependiendo del nivel de endeudamiento, de la posibilidad de ocurrencia de pasivos contingentes o bien de otra causa justificada, este valor podría ser diferente. Así, en caso de sobreendeudamiento, bajo la amenaza de un desastre natural, o debido al envejecimiento de la población, por ejemplo, el centro de las fluctuaciones del RFE podría ser positivo, con la finalidad de ir generando un ahorro. En cambio, en caso de una situación de endeudamiento muy bajo, el centro podría ser temporalmente negativo.

En relación con las cláusulas de escape, se podría plantear que la regla quedara suspendida en caso de que el OG traspasase determinados valores, tanto inferiores como superiores (figura 3). Claro está que si queda suspendida por un importante auge, la discrecionalidad debería dirigirse hacia un mayor ahorro, mientras que si la suspensión fuese por una recesión, entonces la discrecionalidad debería direccionarse hacia un déficit superior. La reactivación de la regla se daría una vez el que OG volviera a los valores dispuestos como umbrales. En caso de que el endeudamiento hubiese superado el valor límite debido a la política contracíclica, entonces se activaría el requerimiento de que el centro de las oscilaciones fuera positivo, generando así el proceso de desendeudamiento. En cambio, en caso de que el auge hubiera generado un ahorro considerado excesivo para afrontar cualquier tipo de contingencia, entonces se podría plantear un déficit de medio plazo hasta que se alcance el nivel de endeudamiento o el monto del fondo soberano deseado. De esta manera, el mismo mecanismo de funcionamiento provee una guía al tratamiento de las desviaciones generadas en los momentos en los que la regla queda desactivada. 
De este modo, la fórmula de la regla sería la siguiente:

\section{$R F E_{t}=x+y O G_{t} ;$ si $|O G|<z$}

donde RFE es el resultado fiscal estructural; " $x$ " es el objetivo de largo plazo o el centro de las fluctuaciones cíclicas del RFE; "y” es el porcentaje permitido o exigido de desviación de corto plazo respecto del objetivo de largo plazo; OG es el output gap; y "z" son los umbrales para la desactivación y reactivación de la regla.

Figura 3: Funcionamiento general de la regla

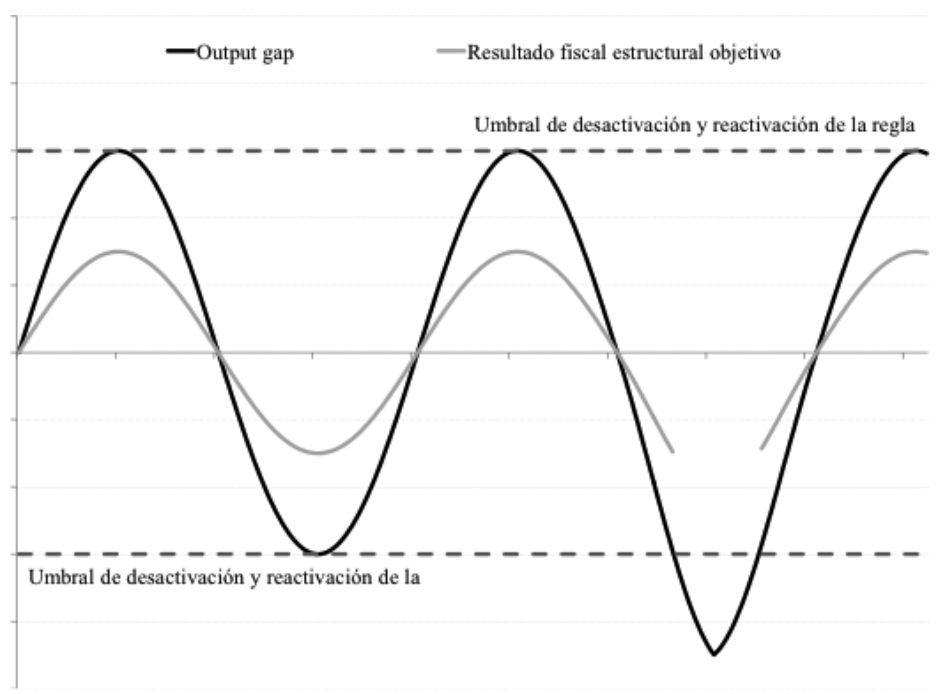

Fuente: Elaboración propia con base en Ter-Minassian (2010) y Schaechter et al. (2012).

El caso de España previo a 2008: un ejercicio de aplicación

El primer paso para aplicar esta regla al caso español es contar con un buen indicador de RFE. Para ello, la primera opción sería tomar el estimado por la Comisión Europea. Sin embargo, si bien esta metodología puede captar parte de los componentes cíclicos de los ingreso y de los gastos, no tiene en cuenta los ingresos extraordinarios por revalorización de activos, a la vez que sufre grandes correcciones ex-post, por lo que basar una decisión de política en este indicador es muy riesgoso.

A cambio, se hará uso del RFE para España estimado por Zack et al. (2014). Como se puede ver en el cuadro 1, este se diferencia significativamente del RFE estimado por la Comisión Europea básicamente por tener en consideración los ingresos públicos extraordinarios producto de la burbuja inmobiliaria y del boom en la construcción. Este indicador identifica que el deterioro fiscal en España comenzó hacia principios de la década del 2000 y ya en 2004 
mostraba un déficit estructural del orden de los 4 puntos porcentuales del PIB. En cambio, para el RFE de la Comisión Europea, al igual que para el RFO, el deterioro fiscal se inicia recién en 2007 y no es hasta 2008, año en el que se inicia la crisis, cuando se observa el primer déficit.

Una vez que se cuenta con un buen indicador de RFE, se procede a establecer los parámetros de la regla. Para el caso de España en los años previos a 2008, se considera que un porcentaje adecuado para el valor objetivo de RFE ("y” en la ecuación 1) es 50\% del OG. Así, por ejemplo, cuando el PIB supere al potencial en un $1 \%$, entonces se exigirá un RFE de $0.5 \%$ del PIB potencial, a lo que habría que sumarle los estabilizadores automáticos para obtener el RFO. En cambio, ante una recesión en la que el PIB se ubique un $2 \%$ por debajo del potencial, la regla misma permitirá incurrir en un déficit estructural del 1\% del PIB potencial, a lo que nuevamente habría que adicionarle los estabilizadores automáticos. En relación con el valor objetivo promedio ("x"), se considera que España no enfrentaba una situación de sobreendeudamiento ni tampoco una necesidad de generar un ahorro a largo plazo $^{8}$. Así, se plantea un equilibrio presupuestario a medio plazo. En cuanto a la cláusula de escape, se establecen los umbrales de suspensión y reactivación de la regla en un valor del OG de 3\% y -3\%, respectivamente. Así, se considera que España no ha experimentado ninguna situación en los años previos a la crisis que justifique la suspensión de la regla.

En definitiva, la fórmula de la regla para España sería del siguiente modo:

$$
R F E_{t}=0+0,5 O G_{t} ; \text { si }|O G|<3 \%
$$

donde RFE es el resultado fiscal estructural y OG es el output gap

A continuación, se realiza el cálculo acerca del ahorro fiscal adicional que hubiera logrado España entre el año 2000 (cuando se incorpora a la Unión Monetaria) y 2008 (el año en el que se inicia de la crisis) en caso de haberse ajustado a una regla de éstas características ${ }^{9}$. Como puede verse en el cuadro 1, estos recursos hubieran alcanzado 48.5\% del PIB. Incluso si se hubiera considerado el RFE estimado por la Comisión Europea, en lugar del calculado por Zack et al. (2014), el ahorro adicional hubiera sido del 25\% del PIB.

Así pues, de haberse aplicado la regla propuesta en España a partir del año 2000, el país hubiese iniciado la crisis con un muy interesante caudal de recursos. Esto, por un lado, hubiera enfriado la economía en los años previos a la crisis, con algún efecto sobre la burbuja inmobiliaria. Pero más importante aún es que se hubiera posibilitado la realización de una política contracíclica más potente una vez iniciada la crisis. En efecto, siguiendo la cláusula de escape propuesta, la regla se hubiera suspendido temporalmente en 2009, ya que el OG fue de $-3.3 \%$ del PIB potencial. De este modo, habría habido vía libre a la discrecionalidad para realizar medidas de impulso a la demanda agregada, por encima de lo permitido por la regla.

En definitiva, este ejercicio muestra la importancia de una regla fiscal diseñada ad hoc (en un contexto de unión monetaria). Para ello es fundamental contar con un indicador efectivo de RFE, que identifique claramente los ingresos extraordinarios. De lo contrario sería muy difícil soportar las presiones para expandir el gasto público cuando la regla exigiera un superávit observado.

\footnotetext{
${ }^{8}$ Un motivo válido para que España se plantee la necesidad de generar un excedente de recursos a largo plazo es el déficit de la seguridad social, especialmente el relacionado con las jubilaciones y pensiones, ante el envejecimiento de la población.

${ }^{9}$ El Anexo II muestra las principales estadísticas económicas de España para el período de análisis.
} 
Tabla 1: Aplicación de la regla fiscal a España (\% del PIB)

\begin{tabular}{ccccccc}
\hline & $\begin{array}{c}\text { Output } \\
\text { Gap }\end{array}$ & $\begin{array}{c}\text { Objetivo de } \\
\text { resultado } \\
\text { fiscal } \\
\text { estructural } \\
\text { según la } \\
\text { regla }\end{array}$ & $\begin{array}{c}\text { Resultado } \\
\text { fiscal } \\
\text { estructural } \\
\text { según Zack } \text { al. } \\
\text { al. }(2014)\end{array}$ & $\begin{array}{c}\text { Ahorro adicional } \\
\text { en caso de } \\
\text { haberse ajustado } \\
\text { a la regla }\end{array}$ & $\begin{array}{c}\text { Resultado fiscal } \\
\text { estructural según } \\
\text { la Comisión } \\
\text { Europea }\end{array}$ & $\begin{array}{c}\text { Ahorro adicional } \\
\text { en caso de } \\
\text { haberse ajustado } \\
\text { a la regla }\end{array}$ \\
\hline 2000 & 3.06 & 1.53 & -2.37 & 3.90 & -2.26 & 3.79 \\
2001 & 3.36 & 1.68 & -2.41 & 4.09 & -2.36 & 4.04 \\
2002 & 2.74 & 1.37 & -2.94 & 4.31 & -1.89 & 3.26 \\
2003 & 2.32 & 1.16 & -3.59 & 4.75 & -1.62 & 2.78 \\
2004 & 2.11 & 1.05 & -4.44 & 5.49 & -1.18 & 2.23 \\
2005 & 2.29 & 1.14 & -4.18 & 5.32 & -0.03 & 1.17 \\
2006 & 2.99 & 1.49 & -4.04 & 5.54 & 0.59 & 0.91 \\
2007 & 3.04 & 1.52 & -4.36 & 5.88 & 0.36 & 1.16 \\
2008 & 1.32 & 0.66 & -8.57 & 9.23 & -5.14 & 5.80 \\
Total & & & & 48.50 & & 25.14 \\
\hline
\end{tabular}

Fuente: Elaboración propia con base en European Commission (2009) y Zack et al. (2014).

Finalmente, una aclaración: lo realizado en esta sección es un ejercicio parcial de política fiscal. Con ello, no se desconoce que las medidas propuestas hubieran tenido efectos sobre otras variables económicas, que podrían haber modificado el escenario. Tampoco se plantea que la política fiscal por sí misma podría haber evitado la crisis. Por el contrario, el ejercicio pretende mostrar únicamente que un comportamiento fiscal diferente en términos cíclicos hubiera sido no solo prudente sino también deseable en pos de la estabilidad real de la economía.

\section{Conclusiones}

El propósito de este artículo ha sido insistir en varias direcciones, muchas de ellas distintas, pero que tienen como objetivo mostrar cómo el manejo de la política económica tiene suficientes aristas para seguir siendo investigadas, ya que de ello depende el buen desempeño de un país, sus empresas y su gente.

En primer lugar, la discusión sobre el papel del Estado en la Economía no es un hecho definido ni contrastado por la evidencia empírica. La crisis de 2008 avala sin duda la intervención, pero lo realmente interesante es enfatizarlo durante períodos de estabilidad y crecimiento, cuando nadie tiene incentivos a arruinar la fiesta y, por lo tanto, las políticas contracíclicas se hacen esperar.

Un segundo punto es la preeminencia que ha tenido el estudio y análisis de la política monetaria y la conclusión sobre la necesidad de seguir reglas predeterminadas. Mientras tanto, por el lado fiscal, si bien se ha debatido mucho en cuanto a las consecuencias de su comportamiento, se ha avanzado poco en la profundización de una regla fiscal. 
Si bien hay evidencia acerca de que la aplicación de una regla fomenta el comportamiento contracíclico de la política fiscal (Bergman y Hutchison, 2015; Grembi et al., 2016; Alberola et al., 2016), no hay tanto consenso en relación al diseño de la regla. Es por esto que, en la primera parte del artículo, luego de mencionar los incentivos existentes para la aplicación de las reglas fiscales, se ha analizado sus opciones de diseño. Así, en primer lugar, se repasaron sus principales trade-offs, entre los que se destacan la disciplina vs. la estabilidad y la flexibilidad vs. la simplicidad, transparencia y cumplimiento de la regla. A continuación, teniendo en cuenta estos trade-offs, se procedió a estudiar las opciones de diseño propiamente dichas. De esta manera, se consideraron las diferentes bases posibles (endeudamiento, gasto, ingresos, resultado fiscal observado o estructural, o alguna combinación de las anteriores) y la necesidad de dotar a la regla de cláusulas de escape claras y transparentes, pero también de plantear la situación que reactivaría automáticamente el funcionamiento de la regla.

Analizadas las opciones de diseño, se realizó una propuesta de regla genérica, la cual tiene como base una combinación entre el RFE y el nivel de endeudamiento, y utiliza al OG como guía para las situaciones de suspensiones temporales y reactivaciones de la regla. Asimismo, para dotarla de mayor flexibilidad, se planteó que el objetivo no sea fijo, sino que fluctúe junto con el OG. Así, la regla no solo exige un superávit observado en el auge, sino también estructural; y lo mismo para el déficit durante las fases de recesión. Cabe destacar que esta mayor capacidad estabilizadora no deteriora el objetivo de solvencia, dado que aboga por un equilibrio a la largo de todo el ciclo.

Esta regla genérica no tiene como objetivo limitar, sino funcionar como una guía para la discrecionalidad y para el buen manejo de las cuentas fiscales agregadas. Es por eso que se deja librado a cada caso en particular la definición de los parámetros de la ecuación (1). De esta forma, cada gobierno puede definir el objetivo de largo plazo (parámetro "x") y corto plazo (parámetro "y"), según sea su propia visión del estado actual de su economía y el objetivo buscado. Del mismo modo, puede definir qué casos son considerados excepcionales como para permitir la desactivación de la regla (y su reactivación automática; parámetro "z").

En este sentido, este trabajo tiene como objetivo central el diseño y propuesta de la regla fiscal, mientras que los trabajos anteriores (Zack et al. 2014) se centraron en analizar y estimar de forma integral una variable central como el RFE, cuyo resumen metodológico se puede ver en el Anexo I. Justamente, el ejercicio para el caso de España (en los años previos a la última crisis) tiene como objetivo ensayar el funcionamiento de la regla a partir del RFE estimado en el trabajo anterior. Para ello, se definen los parámetros "x", "y" y "z", según la situación existente en España hasta 2008 caracterizada por un endeudamiento bajo y un largo ciclo ascendente (ver Anexo II). La aplicación de esta regla hubiera permitido un ahorro equivalente a casi la mitad del PIB de 2008 (en caso de usar el RFE considerando la revalorización de activos) o de la cuarta parte (en caso de no considerar la revalorización de activos).

Desde ya que de haber contado con esos recursos, la respuesta de política de España frente a la crisis que vivió entre 2008 y 2013 (y de la que hoy aun siente las consecuencias) hubiera sido otra. En otras palabras, la regla hubiera permitido un comportamiento fiscal más contracíclico, generando un mayor ahorro en el auge (colaborando así a evitar una mayor burbuja), para poder utilizar esos recursos durante la recesión (colaborando así a evitar una caída tan importante del producto y una tasa de desempleo tan pronunciada).

Finalmente, cabe aclarar que la aplicación de una regla suele ser el resultado de consensos previos acerca de la necesidad de desarrollar una política fiscal más responsable, y no el inicio 
de la misma. Si España estaba a principios del presente siglo en condiciones o no de aplicar una regla de estas características escapa al alcance del presente artículo, aunque no es trivial que haya logrado un superávit fiscal en 2006 y 2007. Del mismo modo, todo país que tenga como objetivo su implementación, deberá analizar previamente si las condiciones están dadas, tanto en el ámbito político como en el social. En otras palabras, la regla difícilmente genere el comportamiento contracíclico de la política fiscal sino, más bien a la inversa, la regla muchas veces es la institucionalización del comportamiento responsable.

\section{Referencias}

Aizenman, J., Gavin, M., \& Hausmann, R. (1996). Optimal Tax Policy with Endogenous Borrowing Constraints. NBER Working Paper, 5558.

Alberola, E., Kataryniuk, I., Melguizo, Á., \& Orozco, R. (2016). Fiscal policy and the cycle in Latin America: the role of financing conditions and fiscal rules. Documentos de trabajo del Banco de España, 1604.

Alesina, A. \& Tabellini, G. (2005). Why is Fiscal Policy Often Procyclical? NBER Working Paper, 11600.

Alesina, A., \& Bayoumi, T. (1996). The Costs and Benefits of Fiscal Rules: Evidence from U. S. States. NBER Working Paper, 5614. https://doi.org/10.3386/w5614

Alt, J. \& Lowry, R. (1994). Divided Government, Fiscal Institutions, and Budget Deficits: Evidence from the States. The American Political Science Review, 88(4), 811-828. http://dx.doi.org/10.2307/2082709

Ayuso-i-Casals, J., González Hernández, D., Moulin, L. \& Turrini, A. (2006). Beyond the SGP - Features and Effects of EU National-Level Fiscal Rules. Preparado para el 2006 Public Finance in EMU European Commission Report, The Role of National Fiscal Rules and Institutions in Shaping Budgetary Outcomes, Bruselas, 24 de noviembre. https://doi.org/10.1057/9780230271791_10

Balassone, F. \& Francese, M. (2004). Cyclical Asymmetry in Fiscal Policy, Debt Accumulation and the Treaty of Maastricht. Banca d'Italia, Temi di Discussione, 531. Barro, R. (1974). Are Government Bonds Wealth? Journal of Political Economy, 82, 1095-1117. https://doi.org/10.1086/260266

Barro, R. (1979). On the Determination of the Public Debt. Journal of Political Economy, 87(5), 940-71. https://doi. org/10.1086/260807

Beetsma, R. \& Uhlig, H. (1999). An Analysis of the Stability and Growth Pact. Economic Journal, 109, 546-571. http:// dx.doi.org/10.1111/1468-0297.00462

Bergman, U. M., \& Hutchison, M. (2015). Economic stabilization in the post-crisis world: Are fiscal rules the answer? Journal of International Money and Finance, 52, 82-101. https://doi.org/10.1016/j.jimonfin.2014.11.014

Blanchard, O., Chouraqui, J-C., Hagemann, R. \& Sartor, N. (1990). The sustainability of fiscal policy: new answers to an old question. OCDE Economic Studies, 15. https://doi.org/10.1787/021121358375

Blanchard, O. \& Perotti, R. (1999). An Empirical Characterization of the Dynamic Effects of Changes in Government Spending and Taxes on Output. NBER Working Paper, 7269. https://doi.org/10.3386/w7269

Bova, E., Carcenac, N., \& Guerguil, M. (2014). Fiscal rules and the procyclicality of fiscal policy in the developing world. IMF Working Paper, 122. https://doi.org/10.5089/9781498305525.001

Bohn, H. \& Inman, R. (1996). Balanced Budget Rules and Public Deficits: Evidence from the U.S. States. NBER Working Paper, 5533. https://doi.org/10.3386/w5533

Buchanan, J. (1997). The balanced budget amendment: Clarifying the arguments. Public Choice, 90(1), 117-138. http://dx.doi.org/10.1023/A:1004969320944

Buchanan, J. \& Wagner, R. (1977). Democracy in Deficit: The Political Legacy of Lord Keynes. Indianapolis: Liberty Fund.

Buti, M., Roeger, W. \& In't Veld, J. (2001). Stabilizing Output and Inflation: Policy Conflicts and Co-operation under a Stability Pact. Journal of Common Market Studies, 39(5), 801-828. http://dx.doi.org/10.1111/1468-5965.00332 
Buti, M. \& Giudice, G. (2002). EMU's Fiscal Rules: What Can and Cannot Be Exported. Preparado para la conferencia Rules-Based Fiscal Policy in Emerging Market Economies, IMF - World Bank, Oaxaca, Mexico, 14-16 de febrero. https://doi.org/10.1057/9781137001573_7

Buti, M., Eijffinger, S. \& Franco, D. (2003). Revisiting the Stability and Growth Pact: grand design or internal adjustment? European Economy, Economic Papers, 180.

Cangiano, M. (1996). Accountability and Transparency in the Public Sector: The New Zeland Experience. IMF Working Paper, 122. https://doi.org/10.5089/9781451854442.001

Catao, L., \& Sutton, B. (2002). Sovereign Defaults: The Role of Volatility, IMF Working Paper, 149. https://doi. org/10.5089/9781451856903.001

Chamorro Narváez, R. A., \& Urrea Bermúdez, A. F. (2016). Effects of fiscal rules on regional public debt sustainability in Colombia. Cuadernos de Economía, 35(SPE67).207-251.

http://dx.doi.org/10.15446/cuad.econ.v35n67.52461

Corsetti, G. \& Roubini, N. (1993). The Design of Optimal Fiscal Rules for Europe After 1992. En F. Torres y F. Giavazzi (Eds.), Adjustment and Growth in the European Monetary Union (pp. 46-82). Cambridge: Cambridge University Press. https://doi.org/10.1017/cbo9780511599231.006

Cuddington, J. (1997). Analysing the sustainability of fiscal deficits in developing countries. Georgetown University, Economics Department. http://inside.mines.edu/ jcudding/papers/Sustain/Sustainability(9.3.99).pdf https://doi. org/10.1596/1813-9450-1784

Cukierman, A. \& Meltzer, A. (1986). A Positive Theory of Discretionary Policy, the Cost of Democratic Government and the Benefits of a Constitution. Economic Inquiry, 24(3), 367-388. http://dx.doi.org/10.1111/j.1465-7295.1986. tb01817.x

Debrun, X., Moulin, L., Turrini, A., Ayuso-i-Casals, J. \& Kumar, M. (2008). Tied to the mast? National fiscal rules in the European Union. Economic Policy, 23(4), 297-362. http://dx.doi.org/10.1111/j.1468-0327.2008.00199.x

Eichengreen, B. (1992). Should the Maastricht Treaty Be Saved?. Princeton Studies in International Finance, 74.

Eichengreen, B. \& von Hagen, J. (1995). Fiscal Policy and Monetary Union: Federalism, Fiscal Restrictions and the No-Bailout Rule. CEPR Discussion Paper, 1247. https://doi.org/10.3386/w5517

European Commission (2000). Public Finance in EMU - 2000. European Economy, 3.

European Commission (2001). Public Finance in EMU - 2001. European Economy, 3.

European Commission (2009). Public Finance in EMU - 2009. European Economy, 5.

Fatás, A., \& Mihov, I. (2006). The macroeconomic effects of fiscal rules in the US states. Journal of Public Economics, 90(1-2), 101-117. http://dx.doi.org/10.1016/j.jpubeco.2005.02.005.

FMI (2009). Crisis and Recovery. World Economic Outlook, abril.

Friedman, M. (1948). A monetary and Fiscal Framework for Economic Stability. The American Economic Review, XXXVIII(3), 245-264. https://doi.org/10.2307/1907322

Friedman, M. (1968). The role of monetary policy. The American Economic Review, LVIII(1), 1-17. https://doi. org/10.1007/978-1-349-24002-9_11

Gavin, M., \& Perotti, R. (2003). Fiscal Policy in Latin America, NBER Macroeconomics Annual, 1997, pp. 11-61. https://doi.org/10.1086/654320

Girouard, N. \& André, C. (2005). Measuring Cyclically-adjusted Budget Balances for OECD Countries. OECD Economics Department Working Papers, 434. https://doi.org/10.1787/787626008442

Grembi, V., Nannicini, T., \& Troiano, U. (2016). Do Fiscal Rules Matter? American Economic Journal: Applied Economics, 8(3), 1-30. https://doi.org/10.1257/app.20150076

Hagemann, R. (1999). The Structural Budget Balance. The IMF’s Methodology. IMF Working Paper, 95. https://doi. org/10.5089/9781451851809.001

Halac, M., \& Yared, P. (2014). Fiscal rules and discretion under persistent shocks. Econometrica, 82(5), 15571614. https://doi.org/10.3982/ecta11207

Hercowitz, Z. \& Strawczynski, M. (2004). Cyclical Ratcheting in Government Spending: Evidence from the OECD. The Review of Economics and Statistics, 86(1), 353-361. http://dx.doi.org/10.1162/003465304323023868 
Inman, R. (1996). Do Balanced Budget Rules Work? U.S. Experience and Possible Lessons for the EMU. NBER Working Paper, 5838. https://doi.org/10.3386/w5838

Jiménez, J. P., \& Ter-Minassian, T. (2016). Política fiscal y ciclo en América Latina: el rol de los gobiernos subnacionales. Serie Macroeconomía del Desarrollo, 173, CEPAL.

Jiménez, J. P. \& Tromben, V. (2006). Política fiscal en países especializados en productos no renovables en América Latina. CEPAL, Serie Macroeconomía del Desarrollo, 46.

Kaminsky, G., Reinhart, C. \& Végh, C. (2004). When it Rains, it Pours: Procyclical Capital Flows and Macroeconomic Policies. NBER Working Paper, 10780. https://doi.org/10.3386/w10780

Kopits, G. (2001). Fiscal Rules: Useful Policy Framework or Unnecessary Ornament? IMF Working Paper, 145. https://doi.org/10.2139/ssrn.2094462

Kopits, G. \& Craig, J. (1998). Transparency in Government Operations. IMF Occasional Paper, 158. https://doi. org/10.5089/9781557756978.084

Kopits, G. \& Symansky, S. (1998). Fiscal Rules. IMF Occasional Paper, 162. https://doi.org/10.5089/9781557757043.084

Kydland, F. \& Prescott, E. (1977). Rules Rather than Discretion: The Inconsistency of Optimal Plans. The Journal of Political Economy, 85(3), 473-491. https://doi.org/10.1086/260580

Lane, P. R. (2002). The Cyclical Behaviour of Fiscal Policy: Evidence from the OECD. Journal of Public Economics, 87(12), 2661-2675. http://dx.doi.org/10.1016/S0047-2727(02)00075-0

Lane, P. R. \& Tornell, A. (1996). Power, growth and the voracity effect. Journal of Economic Growth, 1(2), 213-241. http://dx.doi.org/10.1007/BF00138863

Lane, P. R. \& Tornell, A. (1998). Why aren't Latin American savings rates procyclical? Journal of Development Economics $\mathrm{N}^{\circ}$ 57(1), 185-199. http://dx.doi.org/10.1016/S0304-3878(98)00082-0

Lucas, R. Jr. \& Stockey, N. (1982). Optimal Fiscal and Monetary Policy in an Economy without Capital. The Center for Mathematical Studies in Economics and Management Science Discussion Paper, 532. https://doi. org/10.1016/0304-3932(83)90049-1

Machinea, J. L., Vásquez González, L. \& Zack, G. (2012). La ciclicidad de las políticas públicas latinoamericanas (1995-2010). Madrid: CeALCI - Fundación Carolina.

Manasse, P. (2005). Deficit Limits, Budget Rules, and Fiscal Policy, IMF Working Paper, 120. https://doi. org/10.5089/9781451861396.001

Manasse, P. (2006). Procyclical Fiscal Policy: Shocks, Rules, and Institutions - A View From MARS. IMF Working Paper, 27. https://doi.org/10.5089/9781451862874.001

Nordhaus, V. (1975). The Political Business Cycle. Review of Economic Studies, 42(2), 169-90. https://doi. org $/ 10.2307 / 2296528$

Persson, T. \& Svensson, L. (1989). Why a stubborn conservative would run a deficit: Policy with time-inconsistent preferences. Quarterly Journal of Economics, 104(2), 325-345. https://doi.org/10.2307/2937850

Phelps, E. (1961). The Golden Rule of Accumulation: A Fable for Growthmen. American Economic Review, 51(4), 638-41. https://doi.org/10.1016/b978-0-12-554002-5.50007-8

Poterba, J. M. (1994). State Responses to Fiscal Crises: The Effects of Budgetary Institutions and Politics. Journal of Political Economy, 102(4), 799-821. https://doi.org/10.1086/261955

Rogoff, K. (1990). Equilibrium Political Budget Cycles. The American Economic Review, 80(1), 21-36. https:/doi. org/10.3386/w2428

Sacchi, A., \& Salotti, S. (2015). The impact of national fiscal rules on the stabilisation function of fiscal policy. European Journal of Political Economy, 37, 1-20. https://doi.org/10.1016/j.ejpoleco.2014.10.003

Schaechter, A., Kinda, T., Budina, N. \& Weber, A. (2012). Fiscal Rules in Response to the Crisis-Toward the 'Next-Generation' Rules. A New Dataset. IMF Working Paper, 187. https://doi.org/10.5089/9781475505351.001

Talvi, E. \& Vegh, C. A. (2005). Tax base variability and procyclical fiscal policy in developing countries. Journal of Development Economics, 78(1), 156 - 190. http://dx.doi.org/10.1016/j.jdeveco.2004.07.002

Tanzi, V. (1993). The Political Economy of Fiscal Deficit Reduction. En W. Easterly, C. Rodriguez \& K. Schmidt-Hebbel (Eds.), Public Sector Deficits and Macroeconomic Performance (pp. 513-524). New York: Oxford University Press y World Bank. https://doi.org/10.2307/1061325 
Tapia, H. (2003). Balance estructural del Gobierno central de Chile: análisis y propuestas. Serie macroeconomía del desarrollo, 25, CEPAL.

Taylor, J. B. (2000). Remarks for the Panel Discussion on Recent Changes in Trend and Cycle. Preparado para la conferencia Structural Change and Monetary Policy, Federal Reserve Bank of San Francisco y Stanford Institute for Economic Policy Research, 3-4 de marzo.

Ter-Minassian, T. (2010). Preconditions for a successful introduction of structural fiscal balance-based rules in Latin America and the Caribbean: a framework paper. Inter-American Development Bank Discussion Paper, 157.

Ter-Minassian, T. (2014). Should Latin American countries adopt structural balance-based fiscal rules? Revista de Economía y Estadística, 49(2), 115-143.

Tornell, A. \& Lane, P. R. (1998). Are windfalls a curse? A non-representative agent model of the current account. Journal of International Economics, 44(1), 83-112. http://dx.doi.org/10.1016/S0022-1996(97)00016-0

Velasco, A. (1997). A Model of Endogenous Fiscal Deficits and Delayed Fiscal Reforms. NBER Working Papers, 6336. https://doi.org/10.3386/w6336

Weingast, B., Shepsle, K. \& Johnsen, C. (1981). The Political Economy of Benefits and Costs: A Neoclassical Approach to Distributive Politics. Journal of Political Economy, 89(4), 642-64. https://doi.org/10.1086/260997

Wolf, H. (2005). Volatility: definitions and consequences. En J. Aizenman y B. Pinto (Eds.), Managing Volatility and Crises. A Practitioner Guide (pp. 45-64). Cambridge: Cambridge University Press. https://doi.org/10.1017/ cbo9780511510755.004

Wyplosz, C. (2005). Fiscal Policy: Institutions versus Rules. National Institute Economic Review, 191. https://doi. org/10.1177/0027950105052661

Zack, G. et al. (2014). Towards an Effective Structural Budget Balance for Economic Stability. Hacienda Pública Española / Review of Public Economics, 210-(3/2014): 11-31. http://dx.doi.org/10.7866/HPE-RPE.14.3.1.

\section{Anexo I}

\section{Metodología de cálculo del resultado fiscal estructural (RFE)}

Frente a la necesidad de conocer la situación fiscal de los Estados, se suele acudir al resultado fiscal total o primario. Estos indicadores tienen la desventaja de que reflejan aquellos ingresos y gastos coyunturales y volátiles, que son producto de la fase del ciclo económico. El RFE busca aislar estos componentes cíclicos, dejando únicamente los permanentes o estructurales.

Para ello, en primer lugar, se identifica la fase del ciclo económico mediante el OG. Luego, la relación entre el ciclo y el resultado fiscal se realiza a través de las elasticidades entre la variación en los principales ingresos (impuestos directos sobre las personas físicas y jurídicas, impuestos indirectos y contribuciones a la seguridad social) y los gastos, y la variación del OG (ecuaciones A.2 y A.4). Cada una de estas elasticidades es, a su vez, resultado del producto de dos elasticidades. En el caso de los ingresos, se obtiene en primer lugar la elasticidad entre la variación de la base imponible de cada uno de los impuestos y la variación del OG. Por otro lado, se obtiene la elasticidad entre la recaudación y la base imponible, la cual a veces es estimada y otras veces se hace un supuesto a partir del comportamiento esperado dadas las características del impuesto. Luego, multiplicando estas dos elasticidades, se alcanza la elasticidad que mide la variación en cada uno de los ingresos ante variaciones en el OG (ecuación A.3). La elasticidad total de los ingresos se obtiene mediante un promedio ponderado de las elasticidades de cada impuesto, en función de su participación en el total de la recaudación.

En el caso de los gastos, solo se consideran cíclicos los relacionados con el desempleo. Así, la elasticidad entre los gastos y el OG se obtiene a la partir del producto de la elasticidad entre la variación del gap de desempleo (medida como el cociente entre la tasa de paro observada y la NAWRU) y la variación del OG, y la elasticidad entre la variación del gasto total y la variación del gap de desempleo (ecuación A.5). 
Una vez alcanzados los ingresos y gastos cíclicamente ajustados, se hace la resta para obtener el RFE nominal, que finalmente es dividido por el PIB potencial (ecuación A.1). Esta es la metodología básica de estimación del RFE seguida tanto por la Comisión Europea como por el Fondo Monetario Internacional (Girouard y André, 2005; Hagemann, 1999).

$$
\begin{gathered}
R F^{*}=\frac{T^{*}-G^{*}}{Y^{*}}=\frac{\left(\sum_{i=1}^{4} T_{i}^{*}-G^{*}\right)}{Y^{*}} \\
T_{i}^{*}=T_{i} *\left(\frac{Y^{*}}{Y}\right)^{\varepsilon_{T_{i} ; Y}} \\
\varepsilon_{T_{i} ; Y}=\varepsilon_{B_{i} ; Y} * \varepsilon_{T_{i} ; B_{i}} \\
G^{*}=G *\left(\frac{U^{*}}{U}\right)^{\varepsilon_{G ; U}}=G *\left(\frac{Y^{*}}{Y}\right)^{\varepsilon_{G ; Y}} \\
\varepsilon_{G ; Y}=\varepsilon_{G ; U} * \varepsilon_{U ; Y}=\frac{G_{d}}{G} * \varepsilon_{U ; Y}
\end{gathered}
$$

donde el superíndice “*” indica valor estructural; $\mathrm{RF}$ es el resultado fiscal; $\mathrm{T}_{\mathrm{i}}$ son los ingresos fiscales correspondientes a la categoría "i”; G es el gasto público; es el gasto relacionado con el desempleo; Y es el producto bruto interno; U es la tasa de paro; es la elasticidad de la categoría "i" de ingreso respecto del OG; es la elasticidad de la base imponible de la categoría "i" de ingreso respecto del OG; es la elasticidad del ingreso "i" respecto de su base imponible; es la elasticidad del gasto público respecto del OG; es la elasticidad del gasto público primario respecto del gap de desempleo; y es la elasticidad del desempleo respecto del OG.

Si bien esta metodología es capaz de aislar los componentes cíclicos de los ingresos y los gastos públicos, muchas veces tiene dificultades para identificar el efecto de la sobrevaloración de activos (burbujas), las cuales no siempre se ven completamente reflejadas en el OG. Para ello, se agrega un término a la ecuación A.2 que refleje el efecto del activo sobrevalorado sobre los diferentes ingresos públicos. Así, la ecuación A.2 quedaría reemplazada por la ecuación A.6:

$$
T_{i}^{*}=T_{i} *\left(\frac{Y^{*}}{Y}\right)^{\varepsilon_{T_{i} ;}} *\left(\frac{A^{*}}{A}\right)^{\varepsilon_{T_{i} ; A}}
$$

donde A es el precio del activo sobrevaluado y $\varepsilon_{T} ; A$ es la elasticidad del ingreso "i" en relación al activo sobrevaluado.

En el caso específico de España en los años previos a la última crisis, el RFE se calculó con esta metodología, utilizando a la inversión en vivienda nominal como activo sobrevaluado. Esta variable permite captar tanto la sobrevaluación del precio de las viviendas como el boom en la construcción que afectó las cantidades ${ }^{10}$.

\footnotetext{
${ }^{10}$ Para mayor detalle, ver Zack et al. (2014).
} 


\section{Anexo II:}

Principales estadísticas económicas de España

\begin{tabular}{|c|c|c|c|c|c|c|}
\hline $\begin{array}{c}\text { PIB } \\
\text { (variación } \\
\text { interanual) }\end{array}$ & $\begin{array}{l}\text { Resultado } \\
\text { fiscal } \\
\text { observado } \\
\text { (\% del } \\
\text { PIB) }\end{array}$ & $\begin{array}{l}\text { Ingresos } \\
\text { públicos } \\
\text { (\% del PIB) }\end{array}$ & $\begin{array}{c}\text { Gastos } \\
\text { públicos } \\
(\% \text { del PIB })\end{array}$ & $\begin{array}{c}\text { Deuda pública } \\
\text { (\% del PIB) }\end{array}$ & $\begin{array}{l}\text { Inversión en } \\
\text { vivienda } \\
\text { (\% del PIB) }\end{array}$ & $\begin{array}{c}\text { Tasa de } \\
\text { desempleo } \\
(\% \text { de la PEA })\end{array}$ \\
\hline
\end{tabular}

\begin{tabular}{|c|c|c|c|c|c|c|c|}
\hline 2000 & 5.3 & -1.0 & 38.1 & 39.1 & 58.0 & 8.8 & 11.9 \\
\hline 2001 & 4.0 & -0.5 & 37.9 & 38.5 & 54.2 & 9.1 & 10.6 \\
\hline 2002 & 2.9 & -0.4 & 38.2 & 38.6 & 51.3 & 9.6 & 11.5 \\
\hline 2003 & 3.2 & -0.4 & 37.9 & 38.3 & 47.6 & 10.3 & 11.5 \\
\hline 2004 & 3.2 & 0.0 & 38.6 & 38.7 & 45.3 & 10.9 & 11.0 \\
\hline 2005 & 3.7 & 1.2 & 39.5 & 38.3 & 42.3 & 11.5 & 9.2 \\
\hline 2006 & 4.2 & 2.2 & 40.5 & 38.3 & 38.9 & 12.1 & 8.5 \\
\hline 2007 & 3.8 & 2.0 & 40.9 & 38.9 & 35.5 & 11.7 & 8.2 \\
\hline 2008 & 1.1 & -4.4 & 36.7 & 41.1 & 39.4 & 10.4 & 11.3 \\
\hline 2009 & -3.6 & -11.0 & 34.8 & 45.8 & 52.7 & 8.1 & 17.9 \\
\hline 2010 & 0.0 & -9.4 & 36.2 & 45.6 & 60.1 & 6.9 & 19.9 \\
\hline 2011 & -1.0 & -9.6 & 36.2 & 45.8 & 69.5 & 5.7 & 21.4 \\
\hline 2012 & -2.9 & -10.5 & 37.6 & 48.1 & 85.7 & 4.9 & 24.8 \\
\hline 2013 & -1.7 & -7.0 & 38.6 & 45.6 & 95.4 & 4.1 & 26.1 \\
\hline 2014 & 1.4 & -6.0 & 38.9 & 44.9 & 100.4 & 4.3 & 24.5 \\
\hline 2015 & 3.2 & -5.1 & 38.6 & 43.8 & 99.8 & 4.4 & 22.1 \\
\hline
\end{tabular}

Fuente: Eurostat 